\title{
Evaluation of Poly(Glycidyl Methacrylate) Nanocoating for Chiral Separation with Glu- $\beta$-CD as Chiral Selector in Capillary Electrophoresis
}

\author{
Jin Xu, ${ }^{1}$ Xuecheng Sun, ${ }^{2}$ Xiaodong Sun $\mathbb{D}^{3},{ }^{3}$ and Ye Lu $\mathbb{D}^{4}$ \\ ${ }^{1}$ Department of Neurology, Second Affiliated Hospital of Naval Medical University, Shanghai 200003, China \\ ${ }^{2}$ Research and Development Center of Microelectronics, School of Mechatronic Engineering and Automation, Shanghai University, \\ Shanghai 200444, China \\ ${ }^{3}$ School of Medicine, Shanghai University, Shanghai 200444, China \\ ${ }^{4}$ Department of Anesthesiology, Second Affiliated Hospital of Naval Medical University, Shanghai 200433, China
}

Correspondence should be addressed to Xiaodong Sun; sunxiaodong_cpu@163.com and Ye Lu; smmu1999@126.com

Received 4 July 2021; Accepted 30 July 2021; Published 13 September 2021

Academic Editor: Tingting Hong

Copyright ( 92021 Jin Xu et al. This is an open access article distributed under the Creative Commons Attribution License, which permits unrestricted use, distribution, and reproduction in any medium, provided the original work is properly cited.

In this paper, a capillary coated with poly(glycidyl methacrylate) nanoparticles (PGMA NPs) was prepared and applied to construct a capillary electrophoresis (CE) enantioseparation system with glucosyl- $\beta$-cyclodextrin (Glu- $\beta$-CD) as a chiral selector. The PGMA NP coating can be easily introduced through a simple ring-opening reaction. Two basic drugs were used as models to evaluate the separation performance of the PGMA coating. Under the optimal conditions selected, the separation of the two enantiomers was obtained.

\section{Introduction}

The human body and the two enantiomers show different pharmacodynamics and pharmacokinetic properties, which means controlling the quality of the drug enantiomers is the key factor in the modern pharmaceutical industry. The development of effective and fast chiral drug analysis methods has attracted more and more attention [1-3]. Among various separation techniques, capillary electrophoresis (CE) has proven to be a useful method for chiral drugs due to its high efficiency, simplicity, low consumption, and flexibility of separation methods (e.g., micellar electrokinetic chromatography (MEKC), nonaqueous capillary electrophoresis (NACE), and capillary electrochromatography (CEC)) [4-6]. With further research, the traditional separated CE model has many limitations. Therefore, the induction of new type of materials to modify the inner wall of the capillary and enhance the selectivity and efficiency of $\mathrm{CE}$ has become a commonly used method $[7,8]$.
After being modified by materials such as ionic liquids, nanoparticles, and even phospholipid vesicles, capillaries may have many characteristics, including inhibition of electroosmotic flow (EOF) and local enrichment of chiral selector $[9,10]$. In the past few decades, nanoparticles (NPs) have been used in many fields, including application as column coating for preparing capillary columns $[11,12]$. NPs can provide unique advantages, such as favorable surfaceto-volume ratio, significant mechanical strength, good chemical stability, and easy modification characteristics $[13,14]$. Due to specific physical and chemical properties, they can be easily connected to the capillary to improve the column capacity and efficiency of chiral CE [15-20]. Mayer and Schurig reported the first chiral open tubular CEC system based on Chirasil-Dex- (permethyl- $\beta$-cyclodextrin chemically linked to dimethylpolysiloxane-) modified column for enantioseparation. This method was developed in 1992 [21]. Our group explored an HKUST-1-functionalized CE column for the enantiomeric separation of several racemic 


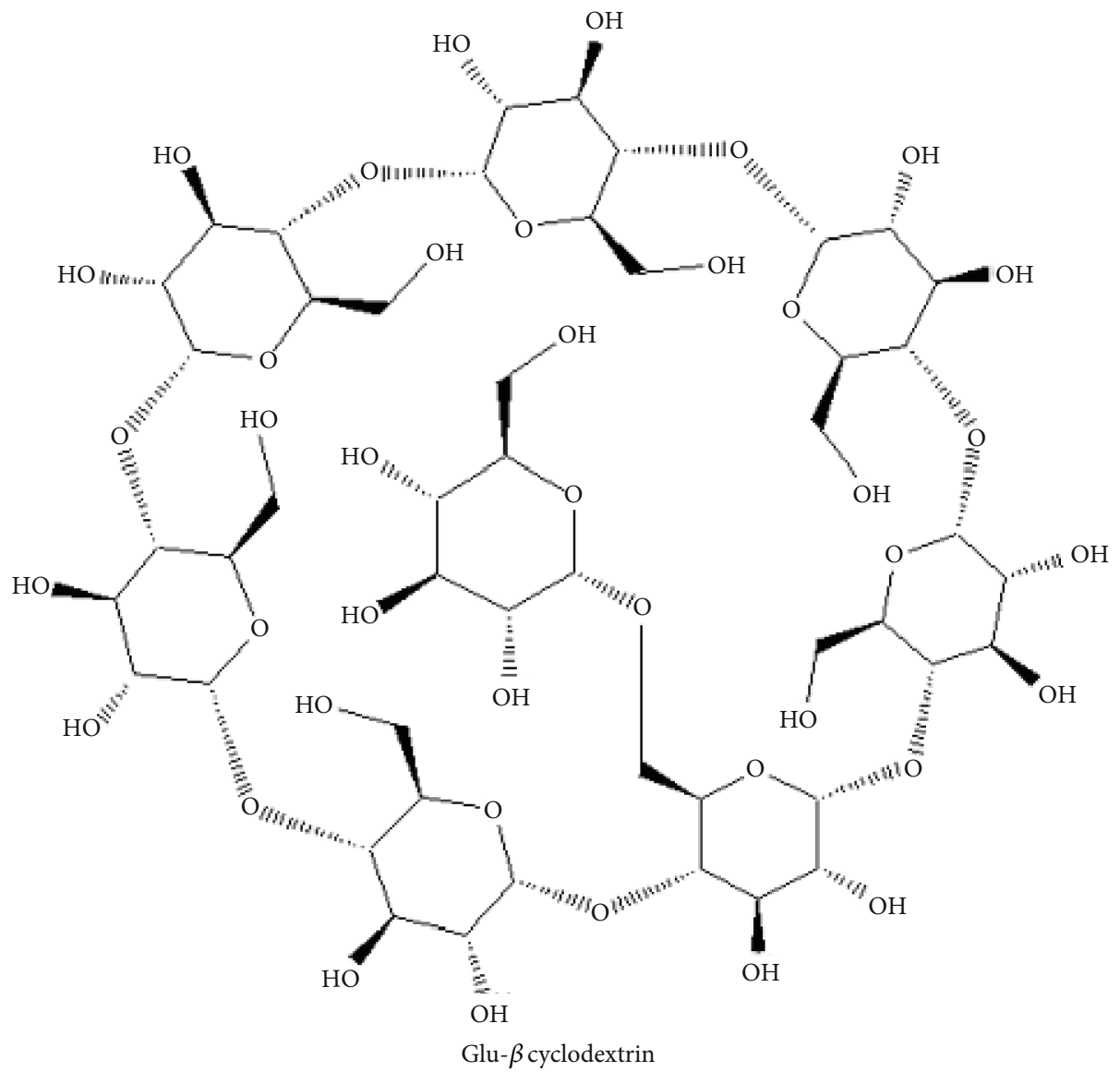

(a)

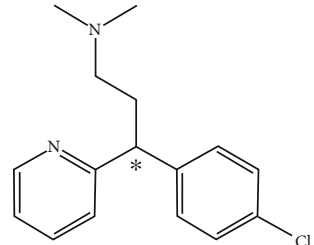

Chlorpheniramine (CHL)

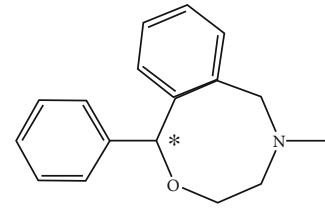

Nefopam (NEF)

(b)

Figure 1: Structure of Glu- $\beta$-CD and racemic drugs.

drugs with $\mathrm{CM}-\beta-\mathrm{CD}$ as the chiral selector in 2019. Compared with the uncoated bare column system, the HKUST1 coating system has significantly improved separation for enantiomers [22].

In this study, poly(glycidyl methacrylate) nanoparticles (PGMA NPs) were immobilized on the inner wall of the capillary through a simple ring-opening reaction [23-26]. Then, Glu- $\beta-C D$ was used as chiral selectors in the PGMA-coated column to construct a Glu- $\beta$-CD/PGMA column separation system. Two essential drugs were selected as models for evaluating enantioselectivity of PGMA nanocoating. Compared with bare column, the new system shows a satisfactory separation performance improvement. The main influencing factors such as chiral selector concentration and background electrolyte (BGE) $\mathrm{pH}$ are systematically studied.

\section{Experimental}

2.1. Materials and Reagents. HPLC grade methanol was purchased from Jiangsu Hanbang Science and Technology Co., Ltd. (Nanjing, China). 3-Aminopropyltriethoxysilane (APTES) and glycidyl methacrylate (GMA) were purchased from Aladdin Chemical Reagent Co., Ltd. (Shanghai, China). Phosphoric acid, sodium hydroxide, hydrochloric acid, and sodium tetraborate decahydrate (all analytical grades) are purchased from Nanjing Chemical Reagent (Nanjing, China). Glu- $\beta$-CD (purity $>99$ ) and racemic drugs (nefopam (NEF) and chlorpheniramine (CHL)) are provided by Jiangsu Food and Drug Administration (Nanjing, China). The structure is shown in Figure 1. All experimental water is ultrapure water. 


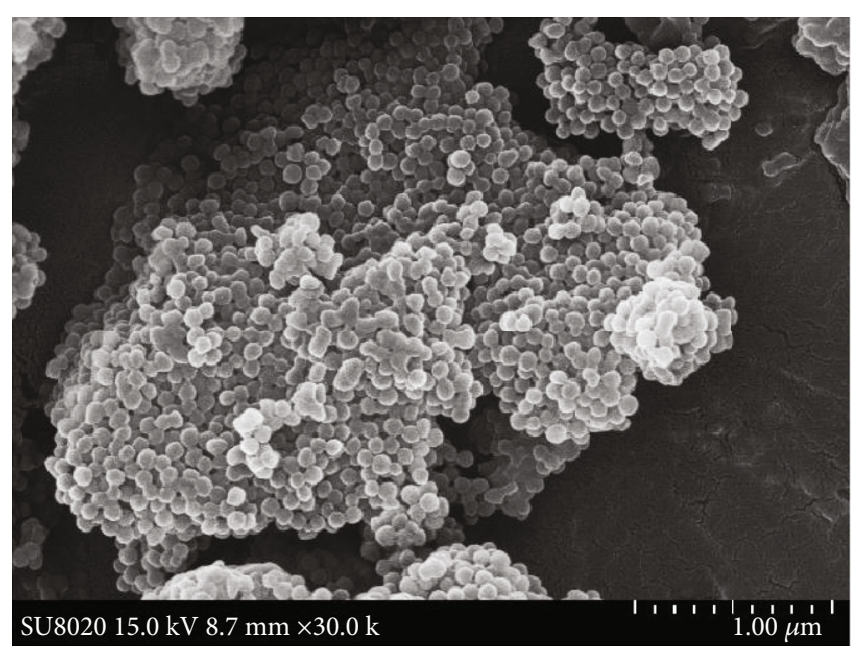

(a)

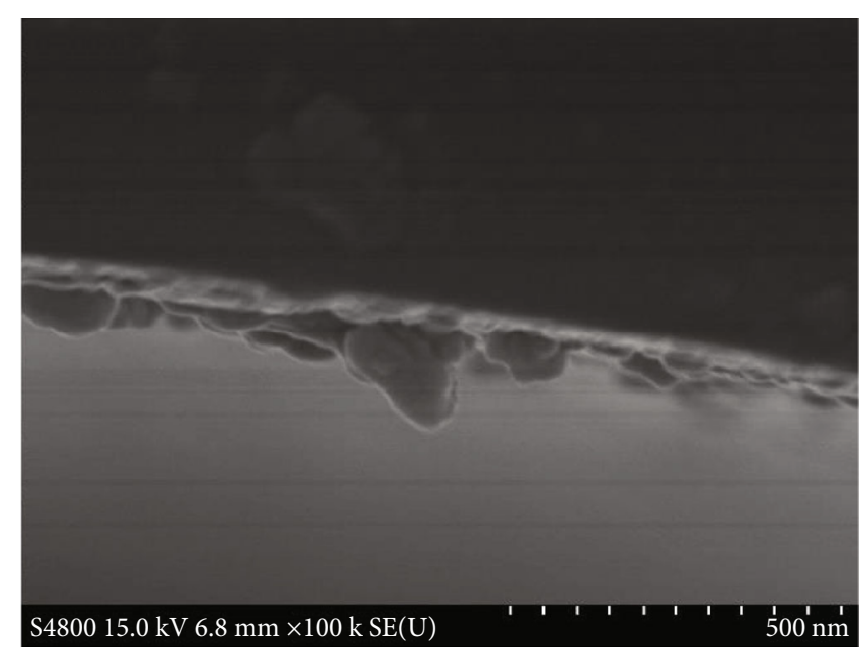

(b)

FIgure 2: SEM of (a) PGMA nanoparticles and (b) PGMA-coated capillary column.

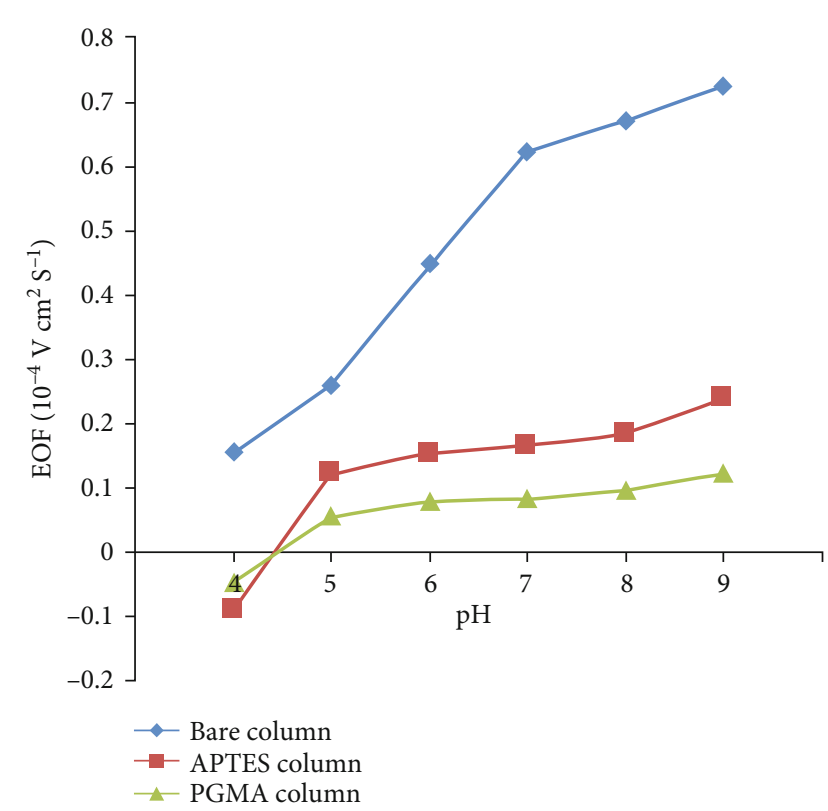

FIGURE 3: Influence of buffer $\mathrm{pH}$ value on the EOF mobility for different columns. Conditions: BGE ( $\mathrm{pH}$ 4.0-9.0); EOF marker, $0.5 \mathrm{mg} / \mathrm{mL}$ thiourea; applied voltage, $15 \mathrm{kV}$.

2.2. Apparatus. A CL1030 CE system (Beijing Huayang Liming Instrumental Co., Beijing, China), equipped with a UV detector $(190-700 \mathrm{~nm})$ and a $30 \mathrm{kV}$ high-voltage power supply, was used to perform all electrophoretic experiments. This article used a capillary column with an inner diameter of $33 \mathrm{~cm}$ (effective length $24.5 \mathrm{~cm}) \times 75$ I.D. The uncoated fused silica capillary tube was provided by Hebei Yongnian Ruifeng Co., Ltd. The morphology of PGMA nanoparticles was characterized by scanning electron microscopy (SEM) (Japan Hitachi SU8010). The PGMA nanocoating in the capillary was characterized by a field emission scanning electron microscope (FESEM, Zeiss Supra, Germany). Between successive injections, flush the capillary column with $0.01 \mathrm{M}$
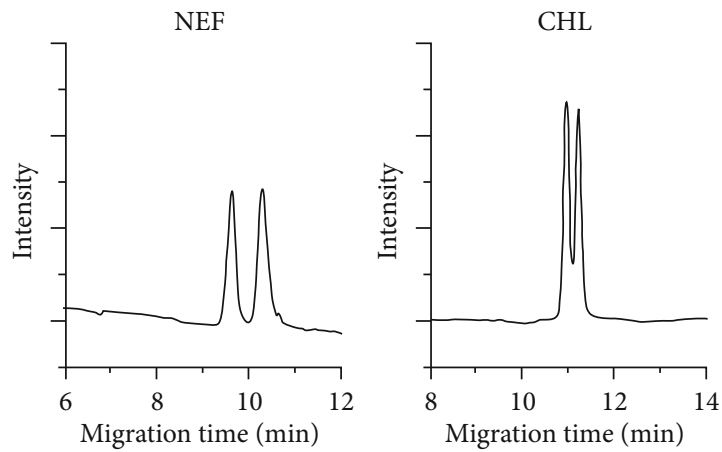

FIgURE 4: Enantioseparation electrophoregrams of NEF and CHL $\left(0.5 \mathrm{mg} \cdot \mathrm{mL}^{-1}\right)$ in PGMA nanocoating with Glu- $\beta-\mathrm{CD}$ as chiral selector. Conditions: $20 \mathrm{mM}$ phosphate buffer; Glu- $\beta-\mathrm{CD}$ concentration, $60 \mathrm{mM}$; $\mathrm{pH}, 6.20$; applied voltage, $15 \mathrm{kV}$.

$\mathrm{NaOH}$ for $60 \mathrm{~s}$, flush with distilled water for $100 \mathrm{~s}$, and then flush with BGE until the baseline is stable. The racemic drug $\left(0.5 \mathrm{mg} \cdot \mathrm{mL}^{-1}\right)$ was dissolved in a dual solvent mixed with methanol and distilled water $(50: 50, v / v)$. Phosphate buffers $(20 \mathrm{mM})$ with various $\mathrm{pH}$ values were prepared for electroosmotic flow (EOF) measurement. Thiourea was chosen as the neutral marker. BGE is freshly prepared by dissolving GLU$\beta$-CD/AL in a buffer solution with a specific $\mathrm{pH}$ value and then adjusted to the desired $\mathrm{pH}$ value by adding a small amount of sodium hydroxide solution using a microsyringe. Before use, all solutions are filtered through a $0.45 \mu \mathrm{m}$ pore membrane filter and degassed by ultrasonic treatment.

\subsection{Preparation of PGMA-Coated Capillary Column}

2.3.1. Synthesis of PGMA Nanoparticles. We followed the methods of Sun et al. [27]. $20 \mathrm{mM} \mathrm{NaHCO}_{3}, 20 \mathrm{mM} \mathrm{Na}_{2} \mathrm{CO}_{3}$, $10 \%(w / v)$ GMA, and $0.3 \%(w / v)$ sodium lauryl sulfate in $100 \mathrm{~mL}$ water were introduced into $250 \mathrm{~mL}$ three-necked circle. After adding $0.2 \%(w / w)$ potassium persulfate, the mixture was stirred at $70^{\circ} \mathrm{C}$ for $12 \mathrm{~h}$. The residual monomer was removed through a syringe (pressure $0.1 \mathrm{MPa}$ ) and 

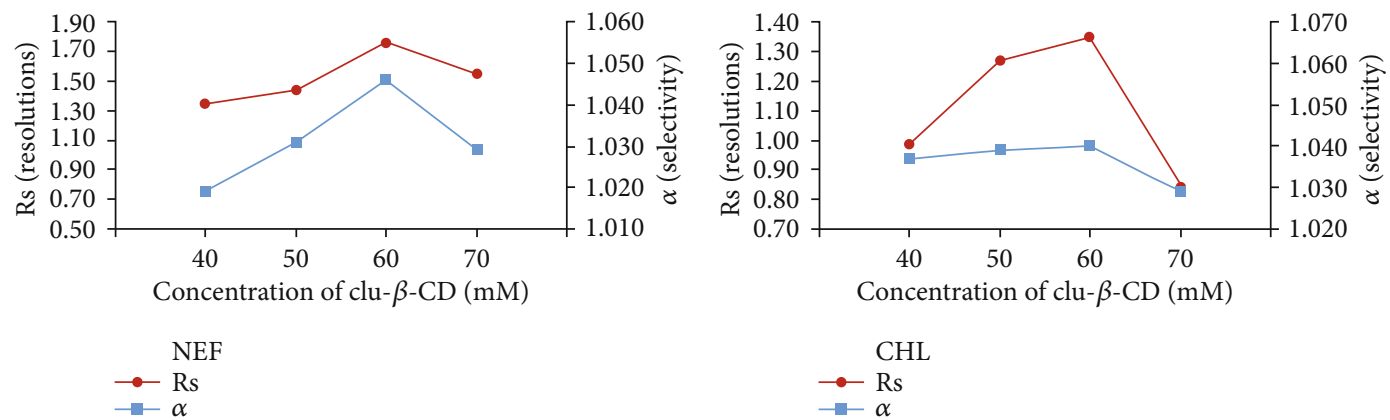

FIGURE 5: Effect of Glu- $\beta$-CD concentration for enantioseparation in PGMA nanocoating system with NEF and $\mathrm{CHL}\left(0.5 \mathrm{mg} \cdot \mathrm{mL}^{-1}\right)$ as $\mathrm{model}^{-}$ drugs. Conditions: $20 \mathrm{mM}$ phosphate buffer with selected Glu- $\beta$-CD concentration $(40-70 \mathrm{mM})$; buffer $\mathrm{pH}, 6.20$; applied voltage, $15 \mathrm{kV}$.
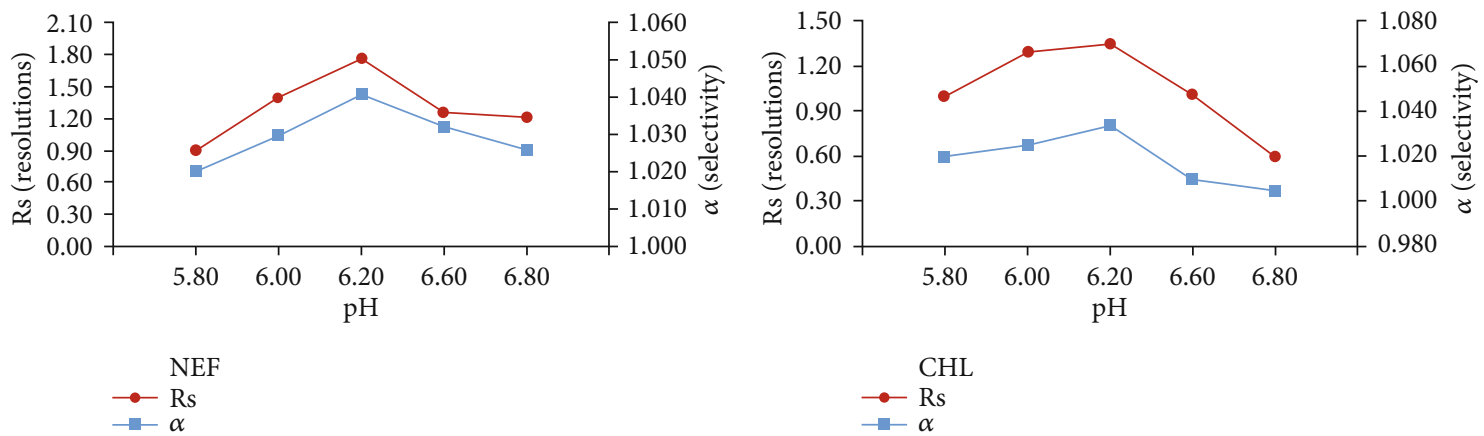

FIGURE 6: Effect of buffer $\mathrm{pH}$ for enantioseparation in PGMA nanocoating system with NEF and CHL $\left(0.5 \mathrm{mg} \cdot \mathrm{mL}^{-1}\right)$ as $\mathrm{model}$ drugs. Conditions: $20 \mathrm{mM}$ phosphate buffer with selected $\mathrm{pH}$ range (5.80-6.80); Glu- $\beta$-CD concentration, $60 \mathrm{mM}$; applied voltage, $15 \mathrm{kV}$.

washed several times. Finally, a white powder was obtained under vacuum at room temperature.

2.3.2. Preparation of $P G M A$ Nanocoating. In our experiment, a $33 \mathrm{~cm}(24.5 \mathrm{~cm}$ effective length $) \times 75 \mu \mathrm{m}$ I.D. fused silica capillary was used. First, wash the capillary with $1.0 \mathrm{M} \mathrm{NaOH}$ (1 h), $\mathrm{H}_{2} \mathrm{O}(30 \mathrm{~min})$, and $1.0 \mathrm{M} \mathrm{HCl}(30 \mathrm{~min})$ in sequence, and then, purge it with methanol for $30 \mathrm{~min}$. After removing the methanol with nitrogen, the capillary was heated in a vacuum oven at $100^{\circ} \mathrm{C}$ for about 1 hour. After cooling to room temperature, the APTES solution $(50 \%, v / v)$ prepared in methanol was pumped through the capillary for $30 \mathrm{~min}$. Then, the capillary column was sealed with rubber and reacted in a water bath at $55^{\circ} \mathrm{C}$ for 12 hours. In order to obtain the PGMA coating, the obtained capillary was rinsed with $0.5 \mathrm{mg} / \mathrm{mL}$ PGMA nanoparticles $(\mathrm{pH} 8.5,50 \mathrm{mM}$ phosphate buffer) for about $30 \mathrm{~min}$ at room temperature. The filled capillary was sealed and allowed to react at $55^{\circ} \mathrm{C}$ for $4 \mathrm{~h}$. Wash the capillary with deionized water to remove excess PGMA nanoparticles. Repeat the above process 3 times.

\section{Results and Discussion}

3.1. Characterization of PGMA Nanoparticles and PGMA Nanocoating. The morphology of PGMA nanoparticles has been clearly characterized by SEM, as shown in Figure 2(a). All PGMA nanoparticles are spherical, with a uniform average diameter and an about $100 \mathrm{~nm}$ narrow particle size distribution. Figure 2(b) shows the SEM image of the inner surface of the PGMA-coated column. Very obvious spherical protrusions can be observed, which indicates that the PGMA nanoparticle coating has been successfully immobilized to the inner wall of the capillary.

3.2. Measurement of EOF. EOF is a key influence due to the fact that it can reflect the ionic situation of capillary inner surface. Here, a range of $\mathrm{pH} 4.0-\mathrm{pH} 9.0$ with thiourea as electroosmotic flow marker in the uncoated capillary, APTSmodified capillary, and PGMA-coated column was investigated. As Figure 3 shows, EOF of the APTS-modified column and PGMA-coated column was negative in $\mathrm{pH} 4$, which is due to the positive charge of the amino group (residual amino for the PGMA-coated column). After immobilization of PGMA NPs, the EOF was lower than that in the APTS column, which was due to the reaction of PGMA with the amino group. It is worth noting that mild EOF change with $\mathrm{pH}$ is favorable for obtaining high stability in migration time.

3.3. Performance of PGMA Nanocoating with Glu- $\beta-C D$ as Chiral Selector. The capillary coating can suppress EOF and reduce the adsorption of analytes, thereby improving separation efficiency. NEF and CHL have been tested to evaluate the modification of PGMA coating. Using $20 \mathrm{mM}$ phosphate buffer and a limited amount of Glu- $\beta-\mathrm{CD}$, the drug is analyzed under optimized separation conditions in the PGMAcoated capillary. From Figure 4, the enantiomeric separation performance of NEF and CHL in PGMA coating system has been obtained. 


\subsection{Optimal Separation Conditions}

3.4.1. Effect of Glu- $\beta$-CD Concentration for Enantioseparation in PGMA Nanocoating System. The concentration of the chiral selector is a key factor affecting the separation of enantiomers. In order to determine the optimal selection agent concentration for the system, a series of concentrations were studied using a $20 \mathrm{mM}$ phosphate buffer solution. As shown in Figure 5, the migration time of racemic drugs all increase with the increase of Glu- $\beta$-CD concentration. The reason for this phenomenon may be the enhanced interaction between the chiral selector and the enantiomer led by local enrichment of Glu- $\beta$-CD in the PGMA nanocoating. And the increase in the viscosity of BGE may be the other reason. As the concentration of the chiral selector increases, Rs and $\alpha$ of the tested drug first increase and then decrease. The maximum value is obtained when the concentration of Glu- $\beta$-CD reaches $60 \mathrm{mM}$. Therefore, $60 \mathrm{mM}$ Glu$\beta$-CD was chosen for the next experiment.

3.4.2. Effect of Buffer $p H$ for Enantioseparation in PGMA Nanocoating System. The $\mathrm{pH}$ of the buffer is an important parameter of the separation system [28]. It determines the degree of ionization of the analyte and the chiral selector and the ion state of the capillary wall. Therefore, optimizing BGE $\mathrm{pH}$ is usually a key strategy for optimizing separation. A $\mathrm{pH}$ range of BGE was studied using a $20 \mathrm{mM}$ phosphate buffer solution containing an optimized concentration of Glu- $\beta$-CD to isolate the selected drugs. As shown in Figure 6 , Rs and $\alpha$ in the two PGMA-coated column systems have the same trend, increasing as the $\mathrm{pH}$ increases from 5.80 to 6.00 . The change trend of resolution gradually decreased from $\mathrm{pH} 6.20$ to 6.80 , and the best separation effect was obtained at 6.20 .

\section{Conclusion}

In this study, a PGMA nanocoating-based enantiomeric separation system with Glu- $\beta-\mathrm{CD}$ as the chiral selector was established to separate enantiomers. In this PGMA-coated column system, the enantiomeric separation results of the $\mathrm{NEF}$ and CHL have been obtained. PGMA nanocoating can achieve satisfactory stability of EOF. In addition, by evaluating influencing factors such as Glu- $\beta$-CD concentration and $\mathrm{BGE} \mathrm{pH}$, the best separation resolution can be obtained with pH 6.20 and $60 \mathrm{mM}$ Glu- $\beta$-CD. This PGMA nanocoating can provide a support to the development of more new types of modification nanomaterials for enantioseparation.

\section{Data Availability}

Due to the confidentiality before public, this manuscript has no associate data.

\section{Conflicts of Interest}

The authors declare that they have no conflicts of interest.

\section{Authors' Contributions}

Jin Xu and Xuecheng Sun contributed equally to this work.

\section{References}

[1] B. Testa, M. Reist, and P. A. Carrupt, "Chirality and drugs," Annales Pharmaceutiques Françaises, vol. 58, p. 239, 2000.

[2] K. H. Engel, "Chirality: an important phenomenon regarding biosynthesis, perception, and authenticity of flavor compounds," Journal of Agricultural and Food Chemistry, vol. 68, no. 38, pp. 10265-10274, 2020.

[3] C. Zhao, X. Qiao, Q. Shao, M. Hassan, and Z. Ma, "Evolution of the lignin chemical structure during the bioethanol production process and its inhibition to enzymatic hydrolysis," Energy \& Fuels, vol. 34, no. 5, pp. 5938-5947, 2020.

[4] Z. Gong, L. Duan, and A. Tang, "Amino-functionalized silica nanoparticles for improved enantiomeric separation in capillary electrophoresis using carboxymethyl- $\beta$-cyclodextrin (CM- $\beta$-CD) as a chiral selector," Microchimica Acta, vol. 182, no. 7-8, pp. 1297-1304, 2015.

[5] X. Sun, J. Guo, T. Yu et al., "A novel coating method for CE capillary using carboxymethyl- $\beta$-cyclodextrin-modified magnetic microparticles as stationary for electrochromatography enantioseparation," Analytical and Bioanalytical Chemistry, vol. 411, no. 6, pp. 1193-1202, 2019.

[6] M. Ramos-Payán, J. A. Ocaña-Gonzalez, R. M. FernándezTorres, A. Llobera, and M. Á. Bello-López, "Recent trends in capillary electrophoresis for complex samples analysis: a review," Electrophoresis, vol. 39, no. 1, pp. 111-125, 2018.

[7] K. Zhang and R. Gao, "Capillary electrochromatography," Methods in Molecular Biology, vol. 52, p. 197, 2001.

[8] Z. Huang, Y. Du, X. Li et al., "A rapid enantioseparation system of capillary electrochromatography modified by electrostatic adsorption with transfersomes," Chirality, vol. 32, no. 1, pp. 98-106, 2020.

[9] L. Yang, E. Guihen, J. D. Holmes, M. Loughran, G. P. O'Sulliva, and J. D. Glennon, "Gold nanoparticle-modified etched capillaries for open-tubular capillary electrochromatography," Analytical Chemistry, vol. 77, no. 6, pp. 1840-1846, 2005.

[10] Z. Liu, Y. Du, and Z. Feng, "Enantioseparation of drugs by capillary electrochromatography using a stationary phase covalently modified with graphene oxide," Microchimica Acta, vol. 184, pp. 583-593, 2018.

[11] Q. Chen, L. Zhu, K. M. Yip et al., "A hybrid platform featuring nanomagnetic ligand fishing for discovering COX-2 selective inhibitors from aerial part of _Saussurea laniceps_ Hand.Mazz," Journal of Ethnopharmacology, vol. 271, p. 113849, 2021.

[12] Q. Chen, L. Zhu, J. Chen et al., "Recent progress in nanomaterial-based assay for the detection of phytotoxins in foods," Food Chemistry, vol. 277, pp. 162-178, 2019.

[13] Q. Zhang, Y. Du, and S. Du, "Evaluation of ionic liquidscoated carbon nanotubes modified chiral separation system with chondroitin sulfate $\mathrm{E}$ as chiral selector in capillary electrophoresis," Journal of Chromatography. A, vol. 1339, pp. 185-191, 2014.

[14] Q. Qu, C. Gu, and X. Hu, "Capillary coated with graphene and graphene oxide sheets as stationary phase for capillary electrochromatography and capillary liquid chromatography," Analytical Chemistry, vol. 84, no. 20, pp. 8880-8890, 2012. 
[15] L. Yang, C. Chen, X. Liu et al., "Use of cyclodextrin-modified gold nanoparticles for enantioseparations of drugs and amino acids based on pseudostationary phase-capillary electrochromatography," Electrophoresis, vol. 31, no. 10, pp. 1697-1705, 2010.

[16] P. Ǩezanka, K. Navrátilová, P. Žvátora et al., “Cyclodextrin modified gold nanoparticles-based open-tubular capillary electrochromatographic separations of polyaromatic hydrocarbons," Journal of Nanoparticle Research, vol. 13, no. 11, pp. 5947-5957, 2011.

[17] X. Li, Y. Du, Z. Feng, X. Sun, and Z. Huang, "A novel enantioseparation approach based on liposome electrokinetic capillary chromatography," Journal of Pharmaceutical and Biomedical Analysis, vol. 145, pp. 186-194, 2017.

[18] L. Li, F. Yang, H. Wang, and X. Yan, "Metal-organic framework polymethyl methacrylate composites for open-tubular capillary electrochromatography," Journal of Chromatography. A, vol. 1316, pp. 97-103, 2013.

[19] Y. Zhang, Y. Zhang, W. Chen et al., "Enantiomeric separation of tryptophan by open-tubular microchip capillary electrophoresis using polydopamine/gold nanoparticles conjugated DNA as stationary phase," Analytical Methods, vol. 9, no. 23, pp. 3561-3568, 2017.

[20] B. Chen and Y. Du, "Evaluation of the enantioseparation capability of the novel chiral selector clindamycin phosphate towards basic drugs by micellar electrokinetic chromatography," Journal of Chromatography. A, vol. 1217, no. 11, pp. 1806-1812, 2010.

[21] S. Mayer, D. Schmalzing, M. Jung, and M. Schleimer, “A chiral test mixture for permethylated $\beta$-cyclodextrin-polysiloxane gas-liquid chromatography phases: the Schurig test mixture," LC GC, vol. 10, no. 10, pp. 782-785, 1992.

[22] X. Sun, Y. Tao, Y. du, W. Ding, C. Chen, and X. Ma, "Metal organic framework HKUST-1 modified with carboxymethyl- $\beta$-cyclodextrin for use in improved open tubular capillary electrochromatographic enantioseparation of five basic drugs," Microchimica Acta, vol. 186, no. 7, pp. 462469, 2019.

[23] X. Sun, Y. Du, S. Zhao, Z. Huang, and Z. Feng, "Enantioseparation of propranolol, amlodipine and metoprolol by electrochromatography using an open tubular capillary modified with $\beta$-cyclodextrin and poly(glycidyl methacrylate) nanoparticles," Microchimica Acta, vol. 186, no. 2, pp. 128-134, 2019.

[24] W. Zhang, S. Piao, and H. Choi, "Facile and fast synthesis of polyaniline-coated poly(glycidyl methacrylate) core-shell microspheres and their electro-responsive characteristics," Journal of Colloid \& Interface Science, vol. 402, pp. 100-106, 2013.

[25] X. Gao, R. Mo, and Y. Ji, "Preparation and characterization of tentacle-type polymer stationary phase modified with graphene oxide for open-tubular capillary electrochromatography," Journal of Chromatography. A, vol. 1400, pp. 19-26, 2015.

[26] S. Zhao, T. Yu, Y. Du et al., "An organic polymer monolith modified with an amino acid ionic liquid and graphene oxide for use in capillary electrochromatography: application to the separation of amino acids, $\beta$-blockers, and nucleotides," Microchimica Acta, vol. 186, no. 9, pp. 636$645,2019$.
[27] X. Sun, W. Ding, C. Chen, T. Yu, Q. Chen, and K. Yang, "Evaluation of poly(glycidyl methacrylate)-coated column for enantioseparation with azithromycin lactobionate and clindamycin phosphate as chiral selectors in capillary electrophoresis," Chromatographia, vol. 84, no. 5, pp. 499-505, 2021.

[28] X. Yao, H. Zheng, Y. Zhang, X. Ma, Y. Xiao, and Y. Wang, "Engineering thiol-ene click chemistry for the fabrication of novel structurally well-defined multifunctional cyclodextrin separation materials for enhanced enantioseparation," Analytical Chemistry, vol. 88, no. 9, pp. 4955-4964, 2016. 